\title{
Stratified scalar field theories of gravitation with self-energy term and effective particle Lagrangian
}

\author{
Diogo P. L. Bragança ${ }^{1,2, a}{ }_{\mathbb{C}}$, José P. S. Lemos ${ }^{1, b}$ \\ ${ }^{1}$ Centro de Astrofísica e Gravitação-CENTRA, Departamento de Física, Instituto Superior Técnico-IST, Universidade de Lisboa-UL, Av. Rovisco \\ Pais 1, 1049-001 Lisbon, Portugal \\ ${ }^{2}$ Present Address: Department of Physics, Stanford Institute of Theoretical Physics-SITP, Stanford University, Stanford, CA 94305, USA
}

Received: 25 April 2018 / Accepted: 19 June 2018 / Published online: 29 June 2018

(c) The Author(s) 2018, corrected publication August 2018

\begin{abstract}
We construct a general stratified scalar theory of gravitation from a field equation that accounts for the self-interaction of the field and a particle Lagrangian, and calculate its post-Newtonian parameters. Using this general framework, we analyze several specific scalar theories of gravitation and check their predictions for the solar system post-Newtonian effects.
\end{abstract}

\section{Introduction}

Newtonian gravitation is a scalar theory in which the gravitational interaction is described by a gravitational scalar field or potential $\Phi$, that satisfies a Poisson equation. It is complemented by Newton's second law of mechanics for the trajectories of particles moving in the gravitational potential $\Phi$.

With the advent of special relativity it became clear that energy and mass are equivalent and so any form of energy, like the energy contained in any physical field, produces gravitational field and also gravitates. The gravitational field, possessing itself gravitational energy, should thus produce additional gravitational field and thus the Poisson law should be modified to contain this field self-energy. A consistent generalization of Newtonian's gravitation accounting for the weight of gravitational self-energy has been performed by several authors, see e.g. [1-4], see also [5] for a discussion of gravitational self-energy terms.

Special relativity also implied that any proposed theory of gravitation should be relativistic. The simplest way is to put Newtonian's gravitation in a relativistic form. Scalar gravitational theories were initiated by Nordström with the gravitational potential being treated as a scalar field on a Minkowski background [6] and then modifying it into a scalar theory in

\footnotetext{
a e-mail: braganca@stanford.edu

be-mail: joselemos@ist.utl.pt
}

a conformal background [7], with its full structure displayed by Einstein and Fokker [8] who showed that it is a covariant scalar theory in a conformally flat space-time, i.e., gravitational effects can be seen as a consequence of having a curved metric generated by a scalar gravitational potential, see also the review by Laue [9]. The idea of conformal theories of gravitation were resurrected by Littlewood [10], whose theory arose the interest of Pirani [11], and further developed by Gürsey [12], Bergmann [13], and Dowker [14]. Several other studies analyzed their properties [15-30]. Reviews, analyses, and modifications of these scalar theories can also be seen in [31-39]. Scalar conformal theories of gravitation are simple, interesting, didactic, but suffer from the problem that due to the conformal character and the coupling of electromagnetism with gravitation, they yield a zero light deflection in the presence of a gravitational field.

An additional interesting set of theories of gravitation that involve a scalar field are the stratified scalar theories. These theories are not purely scalar, they also possess a universal reference frame, where a universal time $t$, and thus a universal vector field, is defined with the space slices composing a stratum that is conformally flat. Einstein was the first to compose such a theory in which the velocity of light is a variable quantity that plays the role of the gravitational potential [40] and was also developed by Abraham [41]. Other stratified theories were composed by Papapetrou [42-44], Yilmaz [45,46], Whitrow and Morduch [47], Page and Tupper [48], Rosen [49], Ni [50,51], and Broekaert [52]. In Ni [50] a review of stratified scalar theories is given. Stratified scalar theories can bypass the light deflection problem. Further, these theories, due to the existence of a preferred vector field, break Lorentz symmetry and thus could be candidates to a fundamental theory where Lorentz symmetry is not essential.

Each of these particular scalar theories, be they conformal or stratified, have a field equation and a particle Lagrangian that together give the particle trajectories in 
the external gravitational field. The theories predict specific values for the classical gravitational tests, see in addition [53-57]. To compare the different theories in these tests, it should then be enough to compare just a few specific parameters of the field equation and of the particle Lagrangian of each theory. They also have different post-Newtonian effects, and we can compare them using the Parametrized Post Newtonian (PPN) formalism [58,59]. The PPN formalism was devised for confronting general relativity and other theories of gravitation with observational data [58-61]. In order to easily compare theories defined by a field equation and particle Lagrangian, a systematic formalism to get PPN parameters from some parameters of the field equation and of particle Lagrangian would be of great help. General relativity, so far the most successful theory, is a tensorial theory of gravitation. But, it is believed that both at the quantum gravity level and at cosmological scales there are corrections to general relativity. On one hand, these corrections bring complexity and give rise to new fields that enter the scene in the same footing as the tensor field of general relativity. Indeed, extensions of general relativity admitting, in addition to the metric tensor field, vector and scalar fields, have been proposed as alternatives theories of gravitation $[58,59]$. On the other hand, general relativity could be emergent from some underlying simpler phenomena, such as atoms of spacetime, perhaps in the form of simple scalar or vectorial fields. Thus, scalar theories of the type just mentioned, or even vectorial theories, can be sought for, although vectorial theories of gravitation modeled in Maxwell electromagnetism suffer from the drawback of admitting negative field energy not admissible for the gravitational field.

In this paper we propose a general stratified scalar theory of gravitation postulating first a field equation that accounts for the self-interaction of the gravitational field and second a Lagrangian for describing particle motion in the gravitational field. Conformal scalar theories of gravitation are also obtained as a specific case of this general stratified scalar theory. We also give a direct method to compute the two PPN parameters that affect the classical solar system tests, namely, Mercury's perihelion precession, light deflection, gravitational redshift, and the Shapiro effect. This method reads the PPN parameters directly from the field equation and the particle Lagrangian. Using specific scalar theories of gravitation we then confront them with experimental data. This method provides a simple, straightforward way to compare between scalar theories of gravitation.

This paper is organized as follows. In Sect. 2, we define the general stratified scalar theory by postulating a gravitational field equation that accounts for self-interaction and a particle Lagrangian that gives the particle motion. In Sect. 3, we calculate the weak field limit and two post-
Newtonian parameters of the theory. In Sect. 4, we use the general scalar theory to compute PPN parameters for specific scalar theories of gravitation. In Sect. 5 we conclude.

\section{Building a general stratified scalar field theory of gravitation with self-interaction}

\subsection{Postulates and equations of the theory}

In a general stratified scalar gravitational theory it is necessary to define an existing prior background structure [50] (see also [58]). Moreover, to build a gravitational theory, one needs to know how a particle moves in a gravitational field and how gravitation is generated by matter. We thus have to (1) define an existing prior background structure, (2) give a general field equation, and (3) give a particle Lagrangian for the particle's trajectories.

The stratified scalar gravitational theory we are going to work with is defined on a background Minkowski spacetime, with line element $d s_{\mathrm{M}}$ given by

$d s_{\mathrm{M}}^{2}=\gamma_{a b} d x^{a} d x^{b}$,

where $\gamma_{a b}$ is the flat spacetime metric, and $a$ and $b$ run from 0 to 3. Note that the coordinates defining Eq. (1) need not be Minkowskian coordinates and so in general $\gamma_{a b}$ need not be $\eta_{a b}=\operatorname{diag}(-1,1,1,1)$. We stratify the theory using a universal time parameter $t$. This parameter is a scalar field satisfying the following equations

$$
\begin{aligned}
\nabla_{b} \nabla_{a} t & =0, \\
\left(\nabla_{a} t\right)\left(\nabla_{b} t\right) \gamma^{a b} & =-1,
\end{aligned}
$$

where $\nabla_{a}$ is the covariant derivative with respect to the metric $\gamma_{a b}$.

To define the field equation for the gravitational scalar field $\Phi$, we generalize the Poisson equation in order to account for a self-interaction of the gravitational field. We then assume that the field equation is given by

$$
\square \Phi=4 \pi G \rho-k \frac{\gamma^{a b} \nabla_{a} \Phi \nabla_{b} \Phi}{c^{2}},
$$

where $\square$ is the d'Alembertian, $\nabla_{a}$ is the covariant derivative, both with respect to the metric $\gamma_{a b}, G$ is Newton's gravitational constant, $\rho$ is the gravitational source density and $k$ is a dimensionless constant.

The gravitational source density $\rho$ is a scalar and thus can be defined in two different ways, namely, $\rho=-T_{a b} u^{a} u^{b}$ where $u^{a}$ is the four-velocity of the source with respect to $\gamma_{a b}$, or $\rho=-\gamma_{a b} T^{a b}$. Although in our work we do not need to specify $\rho$, it is important to note that the Kreuzer 
experiment is not compatible with the first possibility $\rho=$ $-T_{a b} u^{a} u^{b}[50,61]$. This entails that $\rho=-\gamma_{a b} T^{a b}$ is the most realistic choice to be used in Eq. (4). In the case of electromagnetic radiation, we then have $\rho=0$, which means that light does not generate gravitational field. As we shall see, this does not necessarily imply that light is not bent by gravity; this is only the case for conformal scalar theories of gravitation.

Note also that we explicitly include a self-interaction term with coupling constant $k$, but even though different scalar theories of gravity yield a particular $k$, we will let $k$ have a priori any value. See below a derivation of the modified Poisson equation Eq. (4).

We now formulate how test particles behave in the theory. For this, we impose that the particle's trajectories are the geodesics of a metric $g_{a b}$ which itself is generated by the scalar gravitational potential $\Phi$ and the universal time $t$. Thus, we write quite generally [50].

$$
\begin{aligned}
d s^{2} & =g_{a b} d x^{a} d x^{b} \\
& =-(g(\Phi)-f(\Phi)) c^{2} d t^{2}+f(\Phi) \gamma_{a b} d x^{a} d x^{b}
\end{aligned}
$$

where $c$ is the velocity of light, $d t$ is the differential of the universal time $t, \Phi$ is the gravitational potential, and $g$ and $f$ are two scalar functions of $\Phi$. Since $g_{a b}$ in Eq. (5) defines the geodesics it must be considered the physical metric. In general, this physical metric breaks Lorentz symmetries (even though these are preserved in the background metric). This Lorentz symmetry breaking arises also in standard cosmology (e.g. the Cosmic Microwave Background indicates a preferred set of reference frames), and that may indicate that at a fundamental level there must be a breaking of Lorentz symmetries, and as such these stratified theories should not be discarded a priori. Note that light propagates in the null geodesics of this metric, and therefore, in the general case, does not follow straight lines. For the physical metric, the action $S$ for a particle trajectory is then

$S=-m c^{2} \int d \tau$,

where $d \tau=\frac{1}{c} \sqrt{-g_{a b} d x^{a} d x^{b}}$, and $m$ is the mass of the particle. From the standard definition of a Lagrangian $L$, namely,

$S=\int L d x^{0}$

in a given coordinate system $x^{a}$, with $x^{0}$ being some time coordinate, we get, from Eqs. (5), (6), and (7), the following particle Lagrangian,

$$
\begin{aligned}
& L= \\
& -m c \sqrt{(g(\Phi)-f(\Phi)) c^{2}\left(\frac{d t}{d x^{0}}\right)^{2}-f(\Phi) \gamma_{a b} \frac{d x^{a}}{d x^{0}} \frac{d x^{b}}{d x^{0}}} .
\end{aligned}
$$

In a stratified theory, without loss of generality, it is clearly convenient, from (8), that $x^{0}$ should be identified with $t$, $x^{0}=t$. In this case there are further simplifications, namely, $\gamma_{00}=-c^{2}$, and imposing further that the metric is static one can set $\gamma_{0 \alpha}=0$, where $\alpha$ is a spatial index running from 1 to 3. Using these facts, the Lagrangian (8) becomes

$$
L=-m c \sqrt{g(\Phi) c^{2}-f(\Phi) v^{2}}
$$

where the particle's 3-velocity $v$ is defined through the relation

$v^{2}=\gamma_{\alpha \beta} \frac{d x^{\alpha}}{d x^{0}} \frac{d x^{\beta}}{d x^{0}}$.

Since in special relativity the Lagrangian for a particle is [58] $L=-m c \sqrt{c^{2}-v^{2}}$, we see that to get the correct special relativity limit, we have to impose the following condition on the functions $f$ and $g, f(0)=g(0)=1$, where without loss of generality we are assuming that no gravitational field means $\Phi=0$ rather than $\Phi=$ constant. See below a derivation of the particle Lagrangian given in Eq. (9).

It is important to remark that this formalism can be used to study conformally flat theories as well. In fact, to cancel the influence of the universal time $t$, it is sufficient to set $g=f$ in Eq. (8). Choosing a reference frame in which $\gamma_{0 \alpha}=0$, the Lagrangian becomes

$L=-m c \sqrt{f(\Phi)} \sqrt{c^{2}-v^{2}}$

which is simply Eq. (9) with $g=f$, as expected, i.e., it represents a conformal scalar field theory. We will use this fact throughout the paper. Finally, note that, in this case, Eq. (5) shows that light propagates in straight lines.

2.2 Derivation of the Poisson equation with a self-energy term and of the particle Lagrangian

\subsubsection{Derivation of the Poisson equation with a self-energy term}

We can motivate our field equation (4) through the following scheme. In electrodynamics, the electromagnetic energy is stored in the field with a positive energy density $\rho_{\mathrm{EM}}$ given by $\rho_{\mathrm{EM}}=\frac{1}{2}\left(|\boldsymbol{E}|^{2}+|\boldsymbol{B}|^{2}\right)$, where $\boldsymbol{E}$ and $\boldsymbol{B}$ are the electric and magnetic fields, respectively. A similar expression can be obtained for the gravitational field in Newtonian gravitation. Indeed, using Poisson's equation, 
$\nabla^{2} \Phi=4 \pi G \rho$,

one can show that the total gravitational potential energy $E_{\text {grav }}$ in a given volume $V$ can be written as (see e.g. [1]) $E_{\text {grav }}=\frac{1}{2} \int_{V} \rho \Phi \mathrm{d} V=\int_{V}-\frac{|\nabla \Phi|^{2}}{8 \pi G} \mathrm{~d} V$, where $\rho$ is the matter density, $\Phi$ is the gravitational potential, $G$ is Newton's gravitational constant, $\nabla$ is the gradient operator, and an integration by parts has been performed. Therefore, in the Newtonian theory of gravitation, one may define a gravitational field energy density as

$\rho_{\text {grav }} \equiv-\frac{|\nabla \Phi|^{2}}{8 \pi G}$.

It is interesting to note that this is a negative definite energy density. This stems from the fact that gravity in the Newtonian theory is exclusively an attractive force.

Now, we assume that the energy of the gravitational field can also gravitate. Thus, Eq. (12) with Eq. (13) yields $\nabla^{2} \Phi=4 \pi G \rho-\frac{1}{2}|\nabla \Phi|^{2}$. Notice that this approach is not self-consistent (for self-consistent constructions see $[4,16]$ ); nonetheless, this modified Poisson equation is valid to the first post-Newtonian order, which is the order we are interested in for PPN formalism purposes. Moreover, to put in a relativistic setting, and in order to get a Lorentz scalar, we replace the Laplacian in Poisson's equation (12) by the d'Alembertian and generalize the gradient $\nabla$ to the covariant derivative $\nabla_{a}$. Then we get $\square \Phi=4 \pi G \rho-\frac{1}{2} \frac{\gamma^{a b} \nabla_{a} \Phi \nabla_{b} \Phi}{c^{2}}$, where $\gamma_{a b}$ is the Minkowski metric. In order to consider a more general theory, we let the factor $\frac{1}{2}$ that multiplies $\frac{\gamma^{a b} \nabla_{a} \Phi \nabla_{b} \Phi}{c^{2}}$ be undetermined, call it $k$, obtaining thus the sought for equation

$\square \Phi=4 \pi G \rho-k \frac{\gamma^{a b} \nabla_{a} \Phi \nabla_{b} \Phi}{c^{2}}$,

i.e., Eq. (4).

\subsubsection{Derivation of the particle Lagrangian}

We can motivate the definition of our particle Lagrangian (9) from an expression for the energy of a particle in a curved static spacetime.

A static spacetime with metric $g_{a b}$ can be written as

$d s^{2}=g_{a b} d x^{a} d x^{b}=g_{00} d t^{2}+g_{\alpha \beta} d x^{\alpha} d x^{\beta}$,

where Latin indices $a, b$ run from 0 to 3, Greek indices $\alpha, \beta$ correspond to the spatial part of the metric and run from 1 to $3, g_{00}=g_{00}\left(x^{\alpha}\right)$, and in general $g_{\alpha \beta}=g_{\alpha \beta}\left(x^{\alpha}\right)$. We assume asymptotic flatness.

We now calculate the appropriate expression for the observed energy $E$ of a particle measured by a static observer in the metric given by Eq. (15). The four-velocity $v^{a}$ of the particle is $v^{a}=\frac{d x^{a}}{d \tau}$, where $\tau$ is the proper time of the particle, and its four-momentum $p_{a}$ is $p_{a}=m g_{a b} v^{b}$. If an observer has four-velocity $u^{b}$, say, then the total observed energy of the particle measured in the local observer's frame is $E=-p_{b} u^{b}$. Identifying the particle's energy $E$ with the Hamiltonian $H$, we can then find the expression for the Lagrangian $L$.

To start we assume that $g_{\alpha \beta}\left(x^{\alpha}\right)$ is a flat spatial metric, not necessarily Euclidean, afterwards we will relax this assumption. Thus, calling $\gamma_{\alpha \beta}$ a general flat spatial metric, we put

$g_{\alpha \beta}=\gamma_{\alpha \beta}$.

Consider now the metric given in Eq. (15) and consider a coordinate system in which the metric (16) is diagonal, for instance in static spherical coordinates. Then, we have that the particle's four velocity can be written as $v^{a}=$ $\frac{d x^{a}}{d t} \frac{d t}{d \tau}=\frac{d x^{a}}{d t} v^{0}$, where $v^{0}=\frac{d t}{d \tau}$. The spatial components of the velocity $\boldsymbol{v}^{\alpha}$ defined with respect to the time $t$ are $\boldsymbol{v}^{\alpha}=\frac{d x^{\alpha}}{d t}$ and the square of the spatial velocity is $\boldsymbol{v}^{2}=\gamma_{\alpha \beta} \boldsymbol{v}^{\alpha} \boldsymbol{v}^{\beta}$. Then, since $g_{a b} v^{a} v^{b}=-c^{2}$, we get $g_{a b} v^{a} v^{b}=g_{00}\left(v^{0}\right)^{2}+\left(v^{0}\right)^{2} v^{2}=-c^{2}$. We can then solve this equation for $v^{0}$, getting $v^{0}=c \sqrt{\frac{-1}{g_{00}+v^{2}}}$. We want the particle energy measured by an inertial static observer at infinity. Such a static observer has spatial velocity zero, $\boldsymbol{u}^{\alpha}=0$. Since such an observer is also a test particle the above derivation for $v^{0}$ holds, but now we have to replace $v^{0}$ by $u^{0}$ and $\boldsymbol{v}^{\alpha}$ by $\boldsymbol{u}^{\alpha}=0$, an so $\boldsymbol{u}^{2}=0$, and from asymptotically flatness $g_{00}=-c^{2}$. So, $u^{0}=c \sqrt{\frac{-1}{g_{00}}}=1$. Since the metric is diagonal and $\boldsymbol{u}^{\alpha}=0$, the energy expression $E=-p_{b} u^{b}$ simplifies to $E=-m g_{00} v^{0} u^{0}$. Then using the expressions for $v^{0}$ and $u^{0}$ just found we find for the energy the expression

$E=-m g_{00} c \sqrt{\frac{-1}{g_{00}+v^{2}}}$.

We proceed by identifying this energy with the Hamiltonian $H$ of the particle, i.e., $H=E$. Writing the Hamiltonian as a function of the spatial components of the momentum of the particle $p_{\alpha}=m g_{\alpha b} v^{b}$, we obtain

$H=\sqrt{-g_{00} p^{2}-g_{00} m^{2} c^{2}}$,

where here $p^{2}=\gamma^{\alpha \beta} p_{\alpha} p_{\beta}$. This reduces to special relativity when $g_{00}=-c^{2}$. Now, we want the corresponding Lagrangian $L$. Using the Legendre transformation relating $H$ and $L$, namely, $L=\frac{d x^{\alpha}}{d t} p_{\alpha}-H$, together with the Hamilton equation $\frac{d x^{\alpha}}{d t}=\frac{\partial H}{\partial p_{\alpha}}$, we can verify that the particle Lagrangian is 
$L=-m c \sqrt{-g_{00}-v^{2}}$.

We generalize this approach for a non-flat static metric whose spatial part $g_{\alpha \beta}$ can be put in isotropic coordinates. We then write

$g_{\alpha \beta}=f \gamma_{\alpha \beta}$

where $\gamma_{\alpha \beta}$ is a flat metric, not necessarily Euclidean metric, and $f$ is a conformal factor. With this spatial metric, the particle Lagrangian given in Eq. (19) becomes

$L=-m c \sqrt{-g_{00}-f v^{2}}$,

where $v^{2}=\gamma_{\alpha \beta} v^{\alpha} v^{\beta}$. Writing $-g_{00} \equiv g(\Phi) c^{2}$ and $f=$ $f(\Phi)$, for some $\Phi$ and identifying this $\Phi$ with the gravitational potential, we see that the particle Lagrangian in Eq. (21) is the same as

$L=-m c \sqrt{g(\Phi) c^{2}-f(\Phi) v^{2}}$,

i.e., we recover the postulated particle Lagrangian given in Eq. (9).

It is interesting to notice that this particle Lagrangian Eq. (22), or Eq. (9), is the same as the one that comes from the requirement that the trajectory in spacetime is a geodesic of the metric $g_{a b}$. In fact, the particle action in general relativity $S_{\mathrm{GR}}$ is given by $S_{\text {particle GR }}=-m c \int d \tau$, which corresponds to the Lagrangian $L_{\text {particle GR }}=-m c \frac{d \tau}{d t}$. It is immediate to verify that $L_{\text {particle GR }}=L$. Therefore, the particle trajectory in our theory is a geodesic of the metric $d s^{2}=-g(\Phi) c^{2} d t^{2}+f(\Phi) \gamma_{\alpha \beta} d x^{\alpha} d x^{\beta}, \gamma_{\alpha \beta}$ being the spatial flat metric. However, we have derived the Lagrangian Eq. (22), or Eq. (9), without the requirement that a particle follows a geodesic of spacetime and without requiring the covariant divergence of the particle's energy-momentum tensor to vanish. This result is certainly very interesting.

\section{Weak field limit of the theory}

\subsection{Choices of $g$ and $f$}

In order to compare the stratified scalar theory given in Eqs. (4) and (9) with experiment, we take advantage of the Parametrized Post Newtonian (PPN) formalism. To use it, we Taylor expand $f$ and $g$ to second order in $1 / c^{2}$. We define second order terms as having the following factors, $\frac{v^{4}}{c^{4}}, \frac{\Phi v^{2}}{c^{4}}$, or $\frac{\Phi^{2}}{c^{4}}$. The function $g(\Phi)$ is expanded as

$g(\Phi)=1+2 A \frac{\Phi}{c^{2}}+2 B \frac{\Phi^{2}}{c^{4}}$, where $A$ and $B$ are dimensionless constants. Since $f(\Phi)$ appears already multiplied by $v^{2}$ in Eq. (9), it suffices to expand to order $\Phi / c^{2}$, so we write

$f(\Phi)=1-2 C \frac{\Phi}{c^{2}}$

where $C$ is a dimensionless constant. To have the correct Newtonian limit in the Lagrangian (9), we must have $A=1$. From now on, we therefore consider $A=1$.

In order to further understand the physical meaning of $B$ and $C$, we interpret the Lagrangian Eq. (9) as containing an interaction term. For that we should expand the particle Lagrangian given in Eq. (9) to second order and analyze how these parameters affect the interaction part of the Lagrangian. We assume that to second order approximation, we can write our Lagrangian as the sum of a free special relativistic part $L_{\text {particle SR plus an interaction part }}$ $L_{\text {int }}, L=L_{\text {particle SR }}+L_{\text {int }}=-m c^{2} \sqrt{1-\frac{v^{2}}{c^{2}}}+L_{\text {int }}$. Expanding the special relativistic term to second order, we get $L=-m c^{2}+\frac{1}{2} m v^{2}+\frac{1}{8} m \frac{v^{4}}{c^{2}}+L_{\text {int }}$. On the other hand, expanding the particle Lagrangian given by Eq. (9), we get $L=-m c^{2}+\frac{1}{2} m v^{2}\left[1-(2 C+1) \frac{\Phi}{c^{2}}\right]+\frac{1}{8} m \frac{v^{4}}{c^{2}}-$ $m\left[\Phi+\left(B-\frac{1}{2}\right) \frac{\Phi^{2}}{c^{2}}\right]$. Comparing these two equations for $L$ gives the interaction Lagrangian to the relevant order, $L_{\mathrm{int}}=$ $L-L_{\mathrm{SR}}=-m\left[\Phi+\left(B-\frac{1}{2}\right) \frac{\Phi^{2}}{c^{2}}\right]-\frac{1}{2} m v^{2}(2 C+1) \frac{\Phi}{c^{2}}$. Analyzing this equation, we can say, first that $B$ affects the effective gravitational mass of the particle. This is in line with Nordström's interpretation that gravitational mass is affected by the gravitational field itself [7]. Second, that $C$ affects the interaction between the particle's kinetic energy and the gravitational field.

\subsection{Spherical symmetric solution of the field equation}

We have to solve the field equation to obtain the gravitational potential to second order. We solve Eq. (4) in a spherically symmetric vacuum, i.e., $\rho=0$, using spherical coordinates $(r, \theta, \phi)$, obtaining $\frac{d \Phi}{d r}=\frac{G M}{r^{2}+\frac{k G M r}{c^{2}}}$, where $M$ is the gravitational mass of the central body. Up to order $1 / c^{2}$ this yields

$\frac{d \Phi}{d r}=\frac{G M}{r^{2}}+k \frac{(G M)^{2}}{c^{2} r^{3}}$

Integrating Eq. (25) we obtain This corresponds to a potential

$\Phi=-\frac{G M}{r}-k \frac{(G M)^{2}}{2 c^{2} r^{2}}$

with the condition that at infinity $\Phi=0$. 


\subsection{PPN parameters}

We may now substitute Eq. (26) into Eqs. (23) and (24) to get

$g(\Phi)=1-2 \frac{G M}{c^{2} r}+(2 B-k) \frac{(G M)^{2}}{c^{4} r^{2}}$

$f(\Phi)=1+2 C \frac{G M}{c^{2} r}$,

where we kept only the terms to the order previously mentioned.

We want to verify how the parameters $B, C$, and $k$ are related with PPN parameters $\beta$ and $\gamma$ defined in the standard PPN metric on a static, spherically symmetric spacetime in isotropic coordinates by

$$
\begin{aligned}
d s^{2}= & -\left(c^{2}-2 \frac{G M}{r}+2 \beta \frac{(G M)^{2}}{c^{2} r^{2}}\right) d t^{2} \\
& +\left(1+2 \gamma \frac{G M}{c^{2} r}\right) \gamma_{\alpha \beta} d x^{\alpha} d x^{\beta}
\end{aligned}
$$

where $\gamma_{\alpha \beta}$ is a flat 3-metric.

Using the correspondence between $g_{00}$ and $f$, and $g(\Phi)$ and $f(\Phi)$ and comparing with the standard PPN metric Eq. (29), we immediately identify the following relations

$$
\begin{aligned}
A & =1, \\
2 B-k & =2 \beta, \\
C & =\gamma .
\end{aligned}
$$

To be compatible with every solar system test, it is enough to replace $\beta$ and $\gamma$ by the values of general relativity, that is $\beta=\gamma=1$, and guarantee that our theory satisfies the system (30)-(32).

Therefore, a stratified scalar field theory of gravitation that has

- A field equation given in this approximation by Eq. (4),

- A particle Lagrangian given in this approximation by Eq. (9),

- Parameters that satisfy Eqs. (30)-(32) for $\beta=\gamma=1$.

predicts correctly every solar system effect predicted by general relativity.

Moreover, a conformally flat scalar theory of gravitation that has a field equation given in this approximation by Eq. (4), and parameters $A, B, C$, and $k$, that satisfy Eqs. (30)(32) for $\beta=\gamma=1$ would also correctly predict every relativistic solar system effect.

This approach also provides a clean and fast way to compute the PPN parameters $\beta$ and $\gamma$ for most theories of grav- ity, whether stratified scalar field theories or conformally flat scalar theories with only one metric potential.

In the next section, we study consistent theories of gravitation with only one metric potential and calculate for each theory the parameters $B, C$ and $k$ to verify if they predict the correct solar system effects.

\section{Application to scalar field theories of gravitation: Stratified and conformal theories}

\subsection{Page and Tupper theory}

The Page and Tupper theory [48] is a stratified scalar field theory of gravitation and as such is an instance of the set of equations given in Eqs. (4), (5), and (9). In the preferred reference frame, the theory has the following field equation and particle Lagrangian

$$
\begin{aligned}
\Phi & =4 \pi G F\left(\Phi / c_{*}^{2}\right)^{4} \rho \\
L & =-m c \sqrt{F^{2}\left(\Phi / c_{*}^{2}\right) c_{*}(\Phi)^{2}-F^{2}\left(\Phi / c_{*}^{2}\right) v^{2}},
\end{aligned}
$$

respectively, where $\square$ is the d'Alembertian in the Minkowski metric, $\rho$ is the gravitational source density, e.g. $\rho=$ $T_{a b} u^{a} u^{b}$ where $u^{a}$ is the four-velocity of the source, or $\rho=T_{a}^{a}$, and the functions $F\left(\Phi / c_{*}^{2}\right)$ and $c_{*}$ are given by

$$
\begin{aligned}
F & =1-a_{1} \frac{\Phi}{c^{2}}+\left(a_{1} Q+a_{2}\right)\left(\frac{\Phi}{c^{2}}\right)^{2}, \\
c_{*}^{2} & =\frac{c^{2}}{1-Q \frac{\Phi}{c^{2}}+R\left(\frac{\Phi}{c^{2}}\right)^{2}},
\end{aligned}
$$

where $a_{1}, a_{2}, Q$, and $R$, are dimensionless constant parameters in the theory. Here, $c_{*}$ is interpreted as a variable speed of light. Using Eqs. (5), (9), and (34), it is immediate to recover the physical metric of the Page and Tupper theory, and we get for the line element $d s^{2}=F^{2}\left(\Phi / c_{*}^{2}\right)\left(-c_{*}^{2}(\Phi) d t^{2}+d x^{2}+\right.$ $\left.d y^{2}+d z^{2}\right)$.

The field equation Eq. (33) of the Page and Tupper theory corresponds to $k=0$ in Eq. (4). The Lagrangian in Eq. (34) of the Page and Tupper theory corresponds to $g(\Phi) c^{2}=F^{2}\left(\Phi / c_{*}^{2}\right) c_{*}(\Phi)^{2}$ and to $f(\Phi)=F^{2}\left(\Phi / c_{*}^{2}\right)$ in Eq. (9). Expanding to the post-Newtonian order, we get for $g(\Phi)$ and $f(\Phi)$

$$
\begin{aligned}
g(\Phi)= & c^{2}\left[1+\left(Q-2 a_{1}\right) \frac{\Phi}{c^{2}}\right. \\
& \left.+\left(a_{1}^{2}+2 a_{2}+Q^{2}-R\right)\left(\frac{\Phi}{c^{2}}\right)^{2}\right], \\
f(\Phi)= & 1-2 a_{1} \frac{\Phi}{c^{2}},
\end{aligned}
$$


respectively. Looking at Eqs. (23) and (24) this corresponds to

$$
\begin{aligned}
A & =\frac{1}{2} Q-a_{1}, \\
B & =\frac{1}{2}\left(a_{1}^{2}+Q^{2}-R\right)+a_{2}, \\
C & =a_{1} .
\end{aligned}
$$

In order to have the correct Newtonian limit, we must have $A=1$, that is $Q=2 a_{1}+2$. Therefore, the PPN parameters defined in Eq. (29), obeying the relation in Eqs. (30)-(32), are given by

$$
\begin{aligned}
\beta & =\frac{1}{2}\left(a_{1}^{2}+Q^{2}-R\right)+a_{2}, \\
& =\frac{1}{2}\left(5 a_{1}^{2}+8 a_{1}+4-R\right)+a_{2}, \\
\gamma & =a_{1} .
\end{aligned}
$$

The PPN parameter $\beta$ has to be one to account for the solar system tests. We see from Eq. (42) that the Page and Tupper parameters $R$ and $a_{2}$ provide two degrees of freedom, so there are many possible combinations in the theory that give the correct value $\beta=1$. In addition, if in the Page and Tupper theory $a_{1}=1$, then from Eq. (43) the theory has the correct value for $\gamma, \gamma=1$.

\subsection{Ni's Lagrangian-based stratified theory}

This Ni's theory [50] is a stratified scalar field theory of gravitation and as such is an instance of the set of equations given in Eqs. (4), (5), and (9). This theory has a field Lagrangian density which yields the following field equation

$$
\begin{aligned}
& \frac{\partial}{\partial x^{a}}\left[\sqrt{-g} g^{a b} \frac{\partial \Phi}{\partial x^{b}}\right]+2 \pi(-g)^{1 / 2} T^{a b} \frac{\partial g_{a b}}{\partial \Phi} \\
& -\frac{1}{2} \frac{\partial \sqrt{-g} g^{a b}}{\partial \Phi} \frac{\partial \Phi}{\partial x^{a}} \frac{\partial \Phi}{\partial x^{b}}=0, \\
& L=-m c \sqrt{e^{2 \Phi / c^{2}} c^{2}-e^{-2 \Phi / c^{2}} v^{2}} .
\end{aligned}
$$

Using Eqs. (5), (9), and (45), it is immediate to recover the physical metric $g_{a b}$ of this theory, and we get for the line element $d s^{2}=-e^{2 \Phi / c^{2}} d t^{2}+e^{-2 \Phi / c^{2}}\left(d x^{2}+d y^{2}+d z^{2}\right)$.

In a vacuum, this equation simplifies to

$$
\sqrt{-g} g^{a b} \frac{\partial^{2} \Phi}{\partial x^{a} \partial x^{b}}+\frac{1}{2} \frac{\partial\left(\sqrt{-g} g^{a b}\right)}{\partial \Phi} \frac{\partial \Phi}{\partial x^{a}} \frac{\partial \Phi}{\partial x^{b}}=0 .
$$

Using the fact that $\sqrt{-g}=e^{-2 \Phi / c^{2}}$, we obtain

$$
\sqrt{-g} g^{a b}=\operatorname{diag}\left(-e^{-4 \Phi}, 1,1,1\right) .
$$

Therefore, although in general there is a self-interaction term, for the static case (which is the case we are interested in) that term vanishes and Eq. (44) becomes $\nabla^{2} \Phi=0$. This implies that Eq. (44) corresponds to $k=0$ in Eq. (4). The Lagrangian in Eq. (45) corresponds to $g(\Phi)=e^{2 \Phi / c^{2}}$ and to $f(\Phi)=$ $e^{-2 \Phi / c^{2}}$ in Eq. (9). Expanding to the post-Newtonian order, we get for $g(\Phi)$ and $f(\Phi)$

$g(\Phi)=1+\frac{2 \Phi}{c^{2}}+\frac{2 \Phi^{2}}{c^{4}}$,

$f(\Phi)=1-\frac{2 \Phi}{c^{2}}$,

respectively. Looking at Eqs. (23) and (24) this corresponds to

$A=1$,

$B=1$,

$C=1$.

Therefore, the PPN parameters $\beta$ and $\gamma$ defined in Eq. (29), obeying the relations in Eqs. (30)-(32), are given by

$\beta=1$,

$\gamma=1$.

We can conclude that this theory correctly predicts the classical solar system tests.

\subsection{Ni's general conformally flat theory}

This Ni's theory [50] is a conformally flat theory of gravitation and as such is an instance of the set of equations given in Eqs. (4), (5), and (9). The field equation and particle Lagrangian in this theory are given by

$$
\begin{aligned}
\square \Phi & =4 \pi G K(\Phi) \rho, \\
L & =-m c \sqrt{e^{-2 F(\Phi)} c^{2}-e^{-2 F(\Phi)} v^{2}},
\end{aligned}
$$

respectively, where $K(\Phi)=1-p \Phi$ and $F(\Phi)=-\frac{\Phi}{c^{2}}+$ $q \frac{\Phi^{2}}{c^{4}}+\ldots$, with $p$ and $q$ being two dimensionless constants. Note that Nordström's theory [7] is a particular case of this theory. Using Eqs. (5), (9), and (56), it is immediate to recover the physical metric of this theory, and we get for the line element, i.e., the metric, $g_{a b}=e^{-2 F(\Phi)} \gamma_{a b}$, where $\gamma_{a b}$ is a flat metric in Minkowski spacetime, as in Eq. (1).

The field equation Eq. (55) of the theory corresponds to $k=0$ in Eq. (4). The particle Lagrangian in Eq. (56) corresponds to $g(\Phi)=e^{-2 F(\Phi)}$ and to $f(\Phi)=e^{-2 F(\Phi)}$ in Eq. (9). Expanding to the post-Newtonian order, we get for $g(\Phi)$ and $f(\Phi)$ 
$g(\Phi)=1+\frac{2 \Phi}{c^{2}}+(1-q) \frac{2 \Phi^{2}}{c^{4}}$,

$f(\Phi)=1+\frac{2 \Phi}{c^{2}}$,

respectively. Note, that $f(\Phi)$ also gets a $(1-q) \frac{2 \Phi^{2}}{c^{4}}$ in the expansion, but we do not need it in the calculations. Looking at Eqs. (23) and (24) this corresponds to

$B=1-q$,

$C=-1$.

Therefore, the PPN parameters defined in Eq. (29), obeying the relations in Eqs. (30)-(32), are given by

$\beta=1-q$,

$\gamma=-1$.

Therefore, since $\gamma \neq 1$, this theory does not have the correct post-Newtonian form that could explain solar system phenomena.

\subsection{A new conformal scalar theory of gravitation in flat spacetime}

Following Freund and Nambu [16] (see also [4] for the static case), one can build a general self-consistent relativistic scalar theory of gravitation in flat spacetime (see Appendix A).

In this new theory, the vacuum field equation and particle Lagrangian are given by (see Appendix A)

$$
\begin{aligned}
\square \Phi & =-\frac{1}{1-\frac{2 \Phi}{c^{2}}} \frac{1}{c^{2}}\left(\nabla_{c} \Phi\right)\left(\nabla^{c} \Phi\right), \\
L & =-m c^{2} \sqrt{1-\frac{v^{2}}{c^{2}}}\left(1+\frac{\Phi h_{2}(\Phi)}{c^{2}}\right),
\end{aligned}
$$

where $h_{2}$ is a dimensionless function satisfying $h_{2}(0)=1$. Using Eqs. (5), (9), and (64), it is immediate to recover the physical metric of this theory, and we get for the line element, i.e., the metric, $g_{a b}=\left(1+\frac{\Phi h_{2}(\Phi)}{c^{2}}\right)^{2} \gamma_{a b}$, where $\gamma_{a b}$ is a flat metric in Minkowski spacetime, as in Eq. (1).

The field equation Eq. (63) of the theory corresponds to $k=1$ in Eq. (4). The particle Lagrangian in Eq. (64) corresponds to $g(\Phi)=f(\Phi)=\left(1+\Phi h_{2}(\Phi) / c^{2}\right)^{2}$ in Eq. (9). Expanding to the post-Newtonian order, and assuming that we can expand $h_{2}$ to first order in $\Phi$, we get for $g(\Phi)$ and $f(\Phi)$

$$
g(\Phi)=1+\frac{2 \Phi}{c^{2}}+\left(h_{2}^{\prime}(0)+\frac{1}{2}\right) \frac{2 \Phi^{2}}{c^{4}}
$$

$f(\Phi)=1+\frac{2 \Phi}{c^{2}}$

where ' means differentiation with respect to $\Phi$. Note again, that $f(\Phi)$ also gets a $\left(h_{2}^{\prime}(0)+\frac{1}{2}\right) \frac{2 \Phi^{2}}{c^{4}}$ in the expansion, but we do not need it in the calculations. Looking at Eqs. (23) and (24) this corresponds to

$B=h_{2}^{\prime}(0)+\frac{1}{2}$,

$C=-1$,

Therefore, the PPN parameters defined in Eq. (29), obeying the relations in Eqs. (30)-(32), are given by

$\beta=h_{2}^{\prime}(0)$,

$\gamma=-1$.

Even though we have a free parameter $h_{2}^{\prime}(0)$ which could be adjusted to one, this theory always yields $\gamma=-1$, and therefore does not explain all the solar system tests. In order to solve this problem, one could think about relaxing equation Eq. (63) for self-coupling and allow a general $k$. This would only change the expression of $\beta$, which would become $\beta=h_{2}^{\prime}(0)+\frac{1}{2}-\frac{k}{2} ; \gamma$ would still be -1 . This is why it is so challenging to build a relativistic scalar theory of gravitation that respects Lorentz symmetries. Since this theory is very general, the only way to obtain the correct PPN parameters from a scalar field theory in flat spacetime would be to modify the field Lagrangian density by adding for instance a term proportional to $T^{a b}\left(\partial_{a} \Phi\right)\left(\partial_{b} \Phi\right)$. The consequences of this modification cannot be straightforwardly derived with the formalism developed in this paper, since in this case the effective physical metric also depends on $\partial_{a} \Phi$ (that is $f$ and $g$ are functions of $\Phi$ and $\left.\partial_{a} \Phi\right)$ and here we assumed that $f$ and $g$ are only functions of $\Phi$, because this is what was used in the literature. The extension of this model to account for derivative couplings is then left for future work.

\section{Conclusion}

In this paper, we presented a general stratified scalar field theory of gravitation in a Minkowski background. Then, we calculated two post-Newtonian parameters from three general parameters of the theory $B, C$ and $k$, concluding that it is perfectly possible for such a scalar theory to explain the four solar system tests. Finally, we used this general theory to rapidly compute the PPN parameters $\beta$ and $\gamma$ for a set of scalar theories of gravitation to verify if they agree with the experimental tests of gravitation in the solar system. Therefore, with this formalism, one can directly find those two PPN parameters only from the field equation and the particle Lagrangian of a given scalar theory of gravitation. Although 
this is a very efficient method to calculate $\beta$ and $\gamma$ for a given theory, it does not allow one to compute the other PPN parameters. It would be interesting to generalize this approach to efficiently calculate the remaining PPN parameters for scalar theories and verify if it is possible for such a theory to explain every phenomenon predicted by general relativity.

The stratified theories that were analyzed (Page and Tupper's, and Ni's) yielded the correct PPN parameters relevant for solar system tests. One could wonder whether this indicates that they are valid theories, and the answer to that relies in analyzing the remaining PPN parameters. This analysis was done by Nordtvedt and Will [60] and $\mathrm{Ni}$ [50] and the conclusion was that stratified theories cannot account for Earthtide measurements due to the motion of the solar system relative to the preferred frame (defined by the distant stars).

The conformal theories that were analyzed did not yield the correct $\gamma$ parameter even in very general cases. This motivates future work on the analysis of a relativistic scalar theory including a derivative coupling in the Lagrangian, of the type $T^{a b}\left(\partial_{a} \Phi\right)\left(\partial_{b} \Phi\right)$. Such a theory would not have preferred frame effects (it would respect Lorentz symmetries), so if it predicted the correct parameters $\beta$ and $\gamma$ it would not have the problem of Earth-tide measurements.

If such a scalar theory correctly predicts the outcome of every weak field gravity experiment, then we can only rule it out using strong gravity experiment results (e.g. LIGO, neutron star binaries, cosmology). Note also that a scalar theory of gravity is much simpler than general relativity, since it describes gravity with one function instead of ten. In such theories, unlike general relativity, it is generally possible to define a local gravitational energy-momentum tensor, which is always an attractive feature, and is still a problem in general relativity.

Acknowledgements DPLB thanks a grant from Fundação para a Ciência e Tecnologia (FCT), Portugal, through project No. UID/FIS/00099/ 2013. JPSL thanks FCT for the Grant No. SFRH/BSAB/128455/2017, Coordenação de Aperfeiçoamento do Pessoal de Nível Superior (CAPES), Brazil, for support within the Programa CSF-PVE, Grant No. 88887.068694/2014-00.

Open Access This article is distributed under the terms of the Creative Commons Attribution 4.0 International License (http://creativecomm ons.org/licenses/by/4.0/), which permits unrestricted use, distribution, and reproduction in any medium, provided you give appropriate credit to the original author(s) and the source, provide a link to the Creative Commons license, and indicate if changes were made.

Funded by SCOAP $^{3}$.

\section{Appendix A: A new conformal scalar field theory of grav- itation in flat spacetime: criteria and equations}

In this section, following the approach of Freund and Nambu [16] (see also Franklin [4] for the static case) we build a new conformal scalar theory of gravitation in flat Minkowski spacetime from a set of criteria, see Sect. 4.4. These criteria are

1. The spacetime metric is given by

$$
d s^{2}=\gamma_{a b} d x^{a} d x^{b},
$$

where $\gamma_{a b}$ is a flat metric not necessarily of Minkowski form, i.e., not necessarily $\gamma_{a b}=\eta_{a b}=\operatorname{diag}(-1,1,1,1)$.

2. In the Newtonian limit, the field Lagrangian density should be equal to

$$
\mathcal{L}_{\text {Newt }}=-\rho \Phi-\frac{1}{8 \pi G}|\nabla \Phi|^{2} .
$$

3. The general form of the Lagrangian is

$$
\mathcal{L}=\mathcal{L}_{\text {free }}+\mathcal{L}_{\mathrm{m}}+\mathcal{L}_{\text {int }},
$$

where

$\mathcal{L}_{\text {free }}=\frac{h_{1}(\Phi)}{8 \pi G}\left(\partial_{a} \Phi\right)\left(\partial^{a} \Phi\right)$,

with $h_{1}$ being a dimensionless function to be determined which accounts for self-interaction and satisfies $h_{1}(0)=$ $-1, \mathcal{L}_{\mathrm{m}}$ is the matter Lagrangian density, and

$\mathcal{L}_{\text {int }}=\frac{\Phi}{c^{2}} h_{2}(\Phi) T_{\mathrm{m}}$,

with $h_{2}$ a dimensionless free function satisfying $h_{2}(0)=$ 1 , and $T_{\mathrm{m}}$ is the trace of the matter energy-momentum tensor $T_{\mathrm{m}}^{a b}$ defined as $\sqrt{-\gamma} T_{\mathrm{m}}^{a b}=\frac{\delta \mathcal{L}_{\mathrm{m}}}{\delta \gamma_{a b}}, \gamma$ being the determinant of $\gamma_{a b}$, and $\delta$ denotes functional variation.

4. The energy-momentum tensor for the gravitational field is given by Noether's expression

$T_{\text {grav }}^{a b}=\frac{\partial \mathcal{L}_{\text {free }}}{\partial\left(\partial_{a} \Phi\right)} \partial^{b} \Phi-\gamma^{a b} \mathcal{L}_{\text {free }}$,

where $\mathcal{L}_{\text {free }}$ is the free field Lagrangian of Eq. (A.4).

5. The exact free field equation should be of the form

$\square \Phi=\kappa T_{\text {grav }}$,

in order to account explicitly for the self interaction of the field, where $T_{\text {grav }}$ is the trace of $T_{\text {grav }}^{a b}$ and $\alpha$ is a coupling constant to be determined.

6. In a static vacuum, the field equation (A.7) should simplify to

$\nabla^{2} \Phi=-\frac{|\nabla \Phi|^{2}}{c^{2}}$. 
With these requirements in hand, we begin by calculating the expression for $T_{\text {grav }}^{a b}$ using Eqs. (A.4) and (A.6). We then obtain

$T_{\text {grav }}^{a b}=\frac{h_{1}(\Phi)}{4 \pi G}\left[\left(\partial^{a} \Phi\right)\left(\partial^{b} \Phi\right)-\frac{1}{2} \gamma^{a b}\left(\partial_{c} \Phi\right)\left(\partial^{c} \Phi\right)\right]$.

Calculating the trace yields

$T_{\text {grav }}=-\frac{h_{1}(\Phi)}{4 \pi G}\left(\partial_{c} \Phi\right)\left(\partial^{c} \Phi\right)$.

First we fix the constant of proportionality $\kappa$ in Eq. (A.7). In the static, Newtonian limit, where $h_{1}(\Phi)=-1$, we have $T_{\text {grav }}=\frac{1}{4 \pi G}|\nabla \Phi|^{2}$. Therefore, in order to account for Eq. (A.8) one has $\kappa=-\frac{4 \pi G}{c^{2}}$ and the field equation in vacuum is

$\square \Phi=-\frac{4 \pi G}{c^{2}} T_{\text {grav }}$.

Second we determine $h_{1}$. The Euler-Lagrange equation give for the free Lagrangian Eq. (A.4),

$\square \Phi=-\frac{h_{1}^{\prime}(\Phi)}{2 h_{1}(\Phi)}\left(\partial_{c} \Phi\right)\left(\partial^{c} \Phi\right)$.

Replacing Eqs. (A.10) and (A.12) in Eq. (A.11) yields the following differential equation for $h_{1}, h_{1}^{\prime}=-\frac{2}{c^{2}} h_{1}^{2}$, which upon integration gives, considering that $h_{1}(0) \stackrel{c^{2}}{=}-1$,

$h_{1}(\Phi)=-\frac{1}{1-\frac{2 \Phi}{c^{2}}}$.

Thus, using Eq. (A.13) in Eq. (A.10) together with (A.11), or directly in (A.12), we obtain the field equation for the gravitational field $\Phi$ in vacuum,

$\square \Phi=-\frac{1}{c^{2}\left(1-\frac{2 \Phi}{c^{2}}\right)}\left(\partial_{a} \Phi\right)\left(\partial^{a} \Phi\right)$.

The full field equation, i.e., the equation derived taking into account $\mathcal{L}_{\text {free }}$ and $\mathcal{L}_{\text {int }}$ in Eq. (A.3) is then

$$
\begin{aligned}
\square \Phi= & -\frac{1}{c^{2}\left(1-\frac{2 \Phi}{c^{2}}\right)}\left(\partial_{a} \Phi\right)\left(\partial^{a} \Phi\right), \\
& -\frac{4 \pi G}{c^{2}}\left(1-\frac{2 \Phi}{c^{2}}\right)\left(h_{2}+\Phi h_{2}^{\prime}\right) T_{\mathrm{m}} .
\end{aligned}
$$

Finally, we want to find an expression for the matter Lagrangian from $\mathcal{L}_{\mathrm{m}}$ and $\mathcal{L}_{\text {int }}$ in Eq. (A.3). Since we want to compute how particles behave in the theory our matter is represented by a point particle. To simplify the analysis we use Minkowski coordinates, i.e., $\gamma_{a b}=\eta_{a b}, \eta_{a b}=$ $\operatorname{diag}(-1,1,1,1)$. In this case the matter Lagrangian density is the Lagrangian density for a point particle

$$
\begin{aligned}
\mathcal{L}_{\mathrm{m}} & =\rho_{0} \eta_{a b} u^{a} u^{b}, \\
& =m \delta^{3}\left(\boldsymbol{x}-\boldsymbol{x}_{\mathbf{0}}\right) \sqrt{1-\frac{v^{2}}{c^{2}}} \eta_{a b} u^{a} u^{b},
\end{aligned}
$$

where $\rho_{0}$ is the scalar proper mass density, $\boldsymbol{x}$ represents spatial coordinates and $x_{\boldsymbol{0}}$ the spatial position of the particle, and $u^{a}$ is the particle's four-velocity with respect to the metric $\eta_{a b}$. The matter energy-momentum tensor is the energy-momentum tensor for a point particle determined from $T_{\mathrm{m}}^{a b}=\frac{\delta \mathcal{L}_{\mathrm{m}}}{\delta \eta_{a b}}[37]$.

$$
\begin{aligned}
T_{\mathrm{m}}^{a b} & =\rho_{0} u^{a} u^{b}, \\
& =m \delta^{3}\left(\boldsymbol{x}-\boldsymbol{x}_{\mathbf{0}}\right) \sqrt{1-\frac{v^{2}}{c^{2}}} u^{a} u^{b} .
\end{aligned}
$$

This yields the trace

$T_{\mathrm{m}}=-m c^{2} \delta^{3}\left(\boldsymbol{x}-\boldsymbol{x}_{\mathbf{0}}\right) \sqrt{1-\frac{v^{2}}{c^{2}}}$.

Thus, using Eqs. (A.16) and (A.17) for the sum $\mathcal{L}_{\mathrm{m}}+$ $\mathcal{L}_{\text {int }}$ that appears in Eq. (A.3) we have $\mathcal{L}_{\mathrm{m}}+\mathcal{L}_{\text {int }}=$ $-m c^{2} \delta^{3}\left(\boldsymbol{x}-\boldsymbol{x}_{\mathbf{0}}\right) \sqrt{1-\frac{v^{2}}{c^{2}}}\left(1+\frac{\Phi}{c^{2}} h_{2}(\Phi)\right)$, where we have used $\eta_{a b} u^{a} u^{b}=-c^{2}$. Integrating over all space, we get the matter plus the interaction Lagrangian for the particle which we simply call the particle Lagrangian $L, L=$ $\int d^{3} x\left(\mathcal{L}_{\mathrm{m}}+\mathcal{L}_{\text {int }}\right)$, i.e.,

$L=-m c^{2} \sqrt{1-\frac{v^{2}}{c^{2}}}\left(1+\frac{\Phi h_{2}(\Phi)}{c^{2}}\right)$.

The field equation for the gravitational field (A.15) together with the particle Lagrangian (A.19) are the equations of this theory and this is all we need to know in order to calculate the trajectory of particles.

One could make the theory even more interesting by modifying the field Lagrangian density through the addition of a term proportional to $T^{a b}\left(\partial_{a} \Phi\right)\left(\partial_{b} \Phi\right)$.

\section{References}

1. P.C. Peters, Where is the energy stored in a gravitational field? Am. J. Phys. 49, 564 (1981)

2. D. Giulini, Consistently implementing the field self-energy in Newtonian gravity. Phys. Lett. A 232, 165 (1997). arXiv:gr-qc/9605011

3. F.J. Frauendiener, L.B. Szabados, A note on the post-Newtonian limit of quasi-local energy expressions. Class. Quantum Gravity 28, 235009 (2011). arXiv:1102.1867 [gr-qc] 
4. J. Franklin, Self-consistent, self-coupled scalar gravity. Am. J. Phys. 83, 332 (2015). arXiv:1408.3594 [gr-qc]

5. R. Feynman, F. Morinigo, W. Wagner, Feynman Lectures on Gravitation (Addison-Wesley, New York, 1995)

6. G. Nordström, Relativitätsprinzip und Gravitation. Phys. Z. 13, $1126(1912)$

7. G. Nordström, Zur Theorie der Gravitation vom Standpunkt des Relativitätsprinzips. Ann. Phys. 42, 533 (1913)

8. A. Einstein, A.D. Fokker, Die Nordströmsche Gravitationstheorie vom Standpunkt des absoluten Differentialkalküls. Ann. Phys. 44, $321(1914)$

9. M. von Laue, Die Nordströmsche Gravitationstheorie. Jahrb. Radioaktivit. Elektron. 14, 263 (1917)

10. D.E. Littlewood, Conformal transformation and kinematical relativity. Math. Proc. Cambridge Philos. Soc. 14, 90 (1953)

11. F.A.E. Pirani, On the perihelion motion according to Littlewood's equations. Math. Proc. Cambridge Philos. Soc. 51, 535 (1955)

12. F. Gürsey, Gravitation and cosmic expansion in conformal spacetime. Math. Proc. Cambridge Philos. Soc. 49, 285 (1953)

13. O. Bergmann, Scalar field theory as a theory of gravitation I. Am. J. Phys. 24, 38 (1956)

14. J.S. Dowker, A scalar theory of gravitation. Proc. Phys. Soc. 85, 595 (1965)

15. R.U. Sexl, Theories of gravitation. Fortschr. Phys. 16, 269 (1967)

16. P.G.O. Freund, Y. Nambu, Scalar fields coupled to the trace of the energy-momentum tensor. Phys. Rev. 174, 1741 (1968)

17. R.U. Sexl, Universal conventionalism and space-time. Gen. Relativ. Gravit. 1, 159 (1970)

18. S. Deser, L. Halpern, Self-coupled scalar gravitation. Gen. Relativ. Gravit. 1, 131 (1970)

19. T.L.J. Lindén, A scalar field theory of gravitation. Int. J. Theor. Phys. 5, 359 (1972)

20. C.J. Coleman, A simple relativistic theory of gravitation. J. Phys. A 4, 611 (1971)

21. S.L. Shapiro, S.A. Teukolsky, Scalar gravitation: a laboratory for numerical relativity. Phys. Rev. D 47, 1529 (1993)

22. K. Watt, C.W. Misner, Relativistic scalar gravity: a laboratory for numerical relativity. arXiv:gr-qc/9910032

23. S. Calogero, Spherically symmetric steady states of galactic dynamics in scalar gravity. Class. Quantum Grav. 20, 1729 (2003)

24. S. Calogero, G. Rein, Global weak solutions to the NordströmVlasov system. J. Differ. Equ. 204, 323 (2004)

25. F. Ravndal, Scalar gravitation and extra dimensions. arXiv:gr-qc/0405030 (2004)

26. R. Sundrum, Gravity's scalar cousin. arXiv:hep-th/0312212 (2003)

27. F. Girelli, S. Liberati, L. Sindoni, Emergence of Lorentzian signature and scalar gravity. Phys. Rev. D 79, 044019 (2009). arXiv:0806.4239 [gr-qc]

28. N. Deruelle, Nordstrom's scalar theory of gravity and the equivalence principle. Gen. Relativ. Gravit. 43, 3337 (2011). arxiv: 1104.4608 [gr-qc]

29. N. Deruelle, M. Sasaki, Conformal transformations and Nordström's scalar theory of gravity. Progr. Theor. Phys. Suppl. 190, 143 (2011). arXiv:1012.5386 [gr-qc]

30. C. Romero, J.B. Fonseca-Neto, M.L. Pucheu, Conformally flat spacetimes and Weyl frames. Found. Phys. 42, 224 (2012)

31. S.N. Gupta, Einstein's and other theories of gravitation. Rev. Mod. Phys. 29, 334 (1957)

32. A. Schild, Gravitational theories of the Whitehead type and the principle of equivalence, in Evidence for Gravitational Theories, ed. by C. Møller (Academic Press, New York, 1962), p. 69

33. M. Wellner, G. Sandri, Scalar gravitation. Am. J. Phys. 32, 36 (1964)

34. A.L. Harvey, Brief review of Lorentz-covariant scalar theories of gravitation. Am. J. Phys. 33, 449 (1965)
35. G.J. Whitrow, G.E. Morduch, Relativistic theories of gravitation: a comparative analysis with particular reference to astronomical tests. Vistas Astron. 6, 1 (1965)

36. E. Guth, Contribution to the theory of Einstein's geometry as a branch of physics, in Relativity, ed. by M. Carmelli, S. Fickler, L. Witten (Plenum Press, New York, 1970)

37. N.A. Doughty, Lagrangian Interaction: An Introduction to Relativistic Symmetry in Electrodynamics and Gravitation (AddisonWesley, New York, 1990)

38. J.D. Norton, Einstein, Nordström and the early demise of scalar, Lorentz-covariant theories of gravitation. Arch. Hist. Exact Sci. 45, 17 (1992)

39. D. Giulini, What is (not) wrong with scalar gravity? Stud. Hist Philos. Modern Phys. 39, 154 (2008). arXiv:gr-qc/0611100 [gr-qc]

40. A. Einstein, Lichtgeschwindigkeit und Statik des Gravitationsfeldes. Ann. Phys. 38, 355 (1912)

41. M. Abraham, Zur Theorie der Gravitation. Phys. Z. 13, 1 (1912)

42. A. Papapetrou, Eine Theorie des Gravitationsfeldes I. Math. Nachr. 12, 129 (1954)

43. A. Papapetrou, Eine Theorie des Gravitationsfeldes II. Math. Nachr. 12, 1243 (1954)

44. A. Papapetrou, Eine Theorie des Gravitationsfeldes mit einer Feldfunktion. Z. Angew. Phys. 139, 518 (1954)

45. H. Yilmaz, New approach to general relativity. Phys. Rev. 111, 1417 (1958)

46. H. Yilmaz, A theory of gravitation, in Evidence for Gravitational Theories, ed. by C. Møller (Academic Press, New York, 1962), p. 233

47. G.J. Whitrow, G.E. Morduch, General relativity and Lorentzinvariant theories of gravitation. Nature 188, 790 (1960)

48. C. Page, B.O.J. Tupper, Scalar gravitational theories with variable speed of light. Mon. Not. R. Astron. Soc. 138, 67 (1968)

49. N. Rosen, Theory of gravitation. Phys. Rev. D 3, 2317 (1971)

50. W.-T. Ni, Theoretical frameworks for testing relativistic gravity: IV. A compendium of metric theories of gravity and their post Newtonian limits. Astrophys. J. 176, 769 (1972)

51. W.-T. Ni, A new theory of gravity. Phys. Rev. D 10, 2880 (1973)

52. J. Broekaert, A spatially-VSL gravity model with 1-PN limit of GRT. Found. Phys. 38, 409 (2008). arXiv:gr-qc/0405015 [gr-qc]

53. T. Phipps, Mercury's precession according to special relativity. Am. J. Phys. 54, 245 (1986)

54. P.C. Peters, Comment on "Mercury's precession according to special relativity'. Am. J. Phys. 55, 757 (1987)

55. T. Phipps, Response to "Comment on Mercury's precession according to special relativity"' [Am. J. Phys. 55, 757 (1987)]. Am. J. Phys. 55, 75 (1987)

56. W.-T. Ni, Solar-system tests of the relativistic gravity. Int. J. Modern Phys. D 25, 630003 (2016). arXiv:1611.06025 [gr-qc]

57. N.T. Roseveare, Mercury's Perihelion from Le Verrier to Einstein (Oxford University Press, Oxford, 1982)

58. C.M. Will, Theory and Experiment in Gravitational Physics, 2nd edn. (Cambridge University Press, Cambridge, 1993)

59. C.M. Will, The confrontation between general relativity and experiment. Living Rev. Relativ. 17, 4 (2014). arXiv:1403.7377 [gr-qc]

60. K. Nordtvedt, C.M. Will, Conservation laws and preferred frames in relativistic gravity. II. Experimental evidence to rule out preferred-frame theories of gravity. Astrophys. J. 177, 775 (1972)

61. C.M. Will, Active mass in relativistic gravity: theoretical interpretation of the Kreuzer experiment. Astrophys. J. 204, 224 (1976) 